\title{
Tissue Graft Rejection in Mice
}

\section{Survival, Rejection, or Proliferation Across Minor Barriers}

\author{
Jane S. Schultz, Theodore F. Beals, and Roberta DeMott-Friberg \\ Veterans Administration Hospital, Ann Arbor, Michigan 48105 and \\ Departments of Human Genetics and Pathology, \\ University of Michigan, Ann Arbor, Michigan 48109
}

\begin{abstract}
A tissue slice-to-kidney bed grafting system is used to study the mechanism of specific tissue rejection (in this case, rejection of liver tissue) over a series of histocompatibility barriers other than the $\mathrm{H}-2$ barrier. Using the method described, it is possible to obtain a pattern or time-course picture of the immunological process, rather than a mean survival time. It is clear from histological observations of these patterns that, although there are considerable differences in numbers of liver grafts which survive for long periods across the several histocompatibility barriers studied, some grafts in almost every case survive the immunological challenge elicited by the genetic barriers. Grafts of liver tissue are therefore similar, but not identical, in survival patterns to grafts of tumor, ovary, and skin. These studies also indicate that immunological mechanisms controlling rejection of tissue over $\mathrm{H}$ barriers other than $\mathrm{H}-2$ differ from those controlling rejection over the major histocompatibility barrier in the mouse.
\end{abstract}

\section{Introduction}

Skin grafts exchanged between mice differing for the $H-2$ (major histocompatibility) complex survive an average of 8.5 days, whereas grafts exchanged between mice differing at a single minor (non-H-2) histocompatibility locus survive an average of 24 days or more (Klein 1975). Grafts of other tissues-for example, tumors, ovaries, and teeth-may completely overcome the minor histocompatibility barrier, while second-set skin grafts are often rejected as white grafts over these barriers, implicating different immunological mechanisms in rejection (Eichwald et al. 1966). The number of lymphocytes capable of reacting to $\mathrm{H}-2$ antigens in mixed lymphocyte culture or graft-versus-host reaction is also greater than the number capable of reacting to antigens controlled by minor histocompatibility loci (Simonsen 1967), giving further evidence for differences in immunological mechanisms operating in grafts spanning these barriers. Variability of rejection time of individual skin grafts across minor histocompatibility barriers has also been reported, and may be caused by environmental factors, such as antigen dose, allelic combination, and sex of the recipient, as well as by genetic factors (Klein 1967, Lapp and Bliss 1966, McKenzie and Snell 1973, Hildemann et al. 1970). However, Wettstein and 
Haughton (1974) have shown that skin graft rejection over a minor histocompatibility barrier is under genetic control, and that there is a specific $I r$ gene, mapping in the $\mathrm{H}$-2 genetic complex, which regulates responses to at least one minor histocompatibility antigen (Wettstein and Haughton 1977).

The form in which an antigen is administered may influence the immune response to that antigen (Hilgert 1974). If antibodies are formed in the immune response to a particular antigenic form, enhancement of graft (French and Batchelor 1969, Jeekel et al. 1972, Hilgert 1974) or accelerated rejection may result. Thus, final graft outcome is influenced by the site of implantation of the antigens, the quantitative content of antigenic determinants, the form of antigenic presentation, and the genetic difference between donor and host, or a combination of these. $\mathrm{H}$ antigens presented on liver cells or in the form of a liver extract are said to have an enhancing, rather than a rejection-accelerating effect on grafts (Palm and Manson 1965, Mandel et al. 1965). These results can be compared to the rejection-acceleration effect of antigens presented on spleen, thymus, and lung, the neutral reactivity to heart antigens, and the greater enhancing ability of kidney.

To add further to the uncertainty regarding immunological activity in graft rejection across minor histocompatibility barriers, the presence of humoral reactivity has been impossible to demonstrate in animals rejecting skin grafts when donor and recipient differ at these loci. However, sera from grafted animals can prolong or shorten graft survival when passively transferred into recipients grafted with tissue from the same donor. It has been suggested (Klein 1975) that the inability to detect humoral reactivity may result from the presence of the $\mathrm{H}$ antigens on tissues other than those used as a source of target cells for serological tests (lymphocytic cells). Although most attempts to detect humoral activity directed against minor $\mathrm{H}$ antigens were unsuccessful, Goldberg and coworkers (1971) and Cantrell and Hildemann (1973) demonstrated the presence of humoral activity against limited minor histocompatibility systems, using a variety of tests for detection of the humoral response. Recently Zink and Heyner (1977) have shown that antibody to several non-H-2 alloantigens may be elicited by immunization by single-cell suspensions of lymphoid cells, and the presence of these reagents demonstrated by hemagglutination, immunofluorescence, and mixed hemabsorption. Similarly, Baldwin and Cohen (1974) have demonstrated that, despite their inability to elicit more than chronic rejection, weak antigens rapidly immunize hosts, and that the immune response includes a major humoral component that can either accelerate graft rejection or prolong graft survival.

Because of the enormous variability of results obtained in grafting studies of minor histoincompatible animals, and the attendant difficulty in interpretation of these results, studies of minor histocompatibility antigens have been overshadowed by the large number of studies available of tissue rejection by recipients differing from donors for portions of the major histocompatibility complex. However, human analogs of the minor histocompatibility loci of mice are, doubtless, of major importance in clinical organ transplantation, and further elucidation of the immunological mechanisms controlling graft rejection across minor histocompatibility barriers in mice will probably help in understanding organ rejection in humans. It is also possible that certain loci which appear to be minor histocompatibility loci for skin may be expressed on tissues other than skin in a manner rendering them highly accessible to the host immune system, thus causing them to act as major antigens in rejection of grafts of these tissues.

Several reports from this laboratory (Schultz et al. 1976, 1977, Beals et al., in 
preparation, Schultz et al., in preparation) describe the use of a liver slice-to-kidney bed grafting system in the determination of the relative contributions of $\mathrm{H}-2$ and $\mathrm{H}-2$ regional barriers, as well as some non- $\mathrm{H}-2$ barriers, in tissue graft rejection in inbred mouse strains. Using this system, it is possible to study histologically the time course of rejection of relatively uniform small slices of various tissues, and to determine the immunological activity within a graft over a particular genetic barrier, as well as the ultimate fate of the grafted tissue. For a more detailed study of rejection course we have selected liver-to-kidney grafts over five minor locus barriers, which, in our preliminary studies, showed considerable immunological activity or total rejection at 70 days after grafting. We find considerable variability among replicates of these grafts at a single time after grafting. However, the course of immunological activity over any one minor histocompatibility barrier differs from that over other minor histocompatibility barriers and from that found for different alleles at the same minor histocompatibility locus.

\section{Materials and Methods}

Mice. All experimental animals are inbred or congenic mouse strains obtained from the animal colony of the Jackson Laboratory, Bar Harbor, Maine. The following strains were used: B $10 . \mathrm{C}(47 \mathrm{~N}) / \mathrm{Sn}(H$ $\left.7^{b}\right), \mathrm{B} 10.129(5 \mathrm{M}) / \mathrm{nSn}\left(H-1^{b}\right), \mathrm{B} 10 . \mathrm{D} 2(58 \mathrm{~N})\left(H-1^{a}\right)$, B10.LP- $H-3^{b}\left(H-3^{b}\right),\left(\mathrm{B} 10 . \mathrm{D} 2(57 \mathrm{~N}) / \mathrm{Sn}\left(H-\mathcal{8}^{b}\right)\right.$, $\mathrm{B} 10.129(21 \mathrm{M}) / \mathrm{Sn}\left(H-4^{b}\right)$, and C57BL/IUSn (B 10) $\left(H-I^{c}, H-7^{a}, H-3^{a}, H-8^{a}, H-4^{a}\right)$.

Grafting Procedure. The grafting procedure, a modification of the method proposed by Wheeler and coworkers (1966), has been described previously. Briefly, a thin slice of liver tissue is transferred from the anesthetized donor to a recipient kidney site, prepared by removing a small slice from the kidney surface. The recipient skin is sutured and the animal maintained under ordinary laboratory conditions for a predetermined period of time. From four to ten animals of each donor-recipient combination are grafted for each time period studied. After the graft has remained in place for the appropriate time period, the recipient animal is killed, the grafted kidney is removed, and the tissue is fixed, embedded, sectioned, and stained as previously described (Schultz et al. 1976). From three to six slides are prepared and examined histologically for each recipient animal.

Criteria for Evaluation. The slides are evaluated by a consensus of 3 observers and are randomized during the observation process. Slides for replicate animals are evaluated on different days and the primary observation on each slide is made with no knowledge of the donor or recipient of the tissue or the particular experiment to which the sample belongs. In short, all possible measures are taken to keep the observations as objective as possible. Initially a graft is evaluated for rejection or survival by the presence of hepatocytes at the graft site. In addition, the state of immunological reactivity of the host to the graft is judged by the quantity and nature (nodular or scattered) of lymphocytic infiltration, the presence of hemosiderin-laden macrophages, the quantity and physical state of fibrovascular tissue repopulating the graft (young and active or old and collagenized), the distribution of hepatocytes (wellorganized as in a syngeneic graft, active and proliferating, or scattered and degenerating), and the appearance of the scar (newly formed or mature). Technical failures of grafts in this system are minimal and are easily distinguished from rejected grafts.

Although there is a wide variability in the type of immunological reactivity observed within grafts over different barriers, it was possible to establish a scoring system which enables the reader to evaluate quickly the pattern of rejection elicited by each individual immunogenetic barrier. The graft grades and their meanings are as follows: (P), a proliferating graft with more than 4 layers of hepatocytes with or without cellular immunological activity; (S), a syngeneic-like graft with 3 to 4 well-organized layers of hepatocytes with little or no cellular immunological activity; (C-1) a healthy graft with a few lymphocytes concentrated or in small nodules at the graft-bed interface; $(\mathrm{C}-2)$, a graft containing less healthy, poorly organized hepatocytes with a considerable number of lymphocytes scattered throughout the graft; (C-3) degenerating scattered hepatocytes, copious lymphocytic infiltration and fibrovascular tissue; (C-4), lymphocytes present in large nodules with a few scattered hepatocytes in a young scar; (C-5), fibrovascular tissue with some collagen (young scar), a few lymphocytes present and no 
hepatocytes; (C-6), mature (contracted) scar, lymphocytes have receded, the appearance of this graft is indistinguishable from the appearance of an acutely rejected graft (over an $\mathrm{H}-2$ barrier).

\section{Results}

Figures 1-3 are photomicrographs of grafts illustrating three of the grades described above.

Summarized results of all graft series spanning minor histocompatibility barriers can be found in Table 1 and Figure 4. There is immunological activity at all postgraft periods in grafts spanning an $\mathrm{H}-7$ barrier, with most grafts rejected at 70 and 84 days after grafting. Three alleles of the $\mathrm{H}-1$ histocompatibility barrier were studied and results are presented in Table 1 . In the $H-1^{c} \rightarrow H-1^{b}$ combination, there are surviving grafts as late as 56 days after grafting, but rejection is complete at 70 days after grafting. $H-1^{a} \rightarrow H-1^{c}$ and $H-1^{b} \rightarrow H-1^{c}$ grafts appear to behave in a manner similar to syngeneic grafts. However, there are some cases in which these grafts are proliferating. $H-1^{b} \rightarrow H-1^{a}$ liver slice-to-kidney bed grafts are surviving and healthy at 70 days. The reciprocal combination, $H-1^{a} \rightarrow H-1^{b}$, shows slightly more immunological activity at 70 days. This result is at variance with available skin

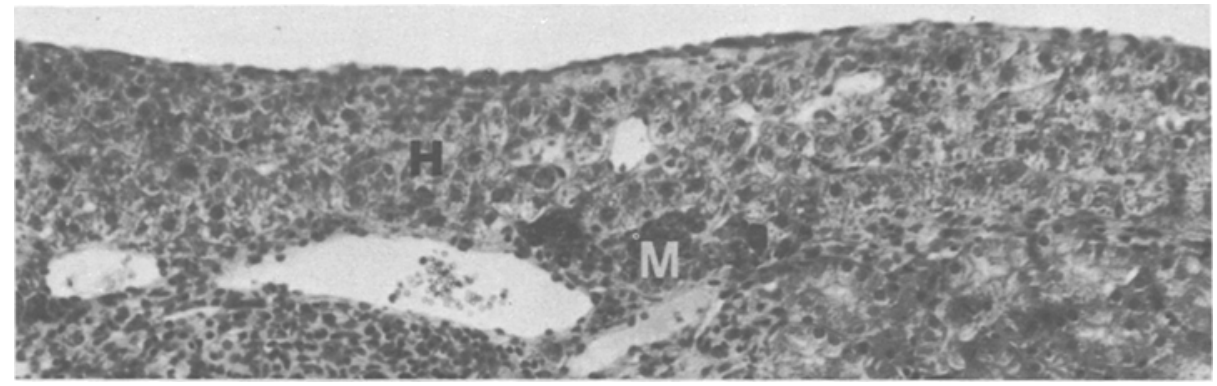

Fig. 1. B 10.129(21M) 42 days after grafting appears as a syngeneic graft. Hepatocytes $(H)$. Hemosiderin-laden macrophages $(M) .(200 \times)$

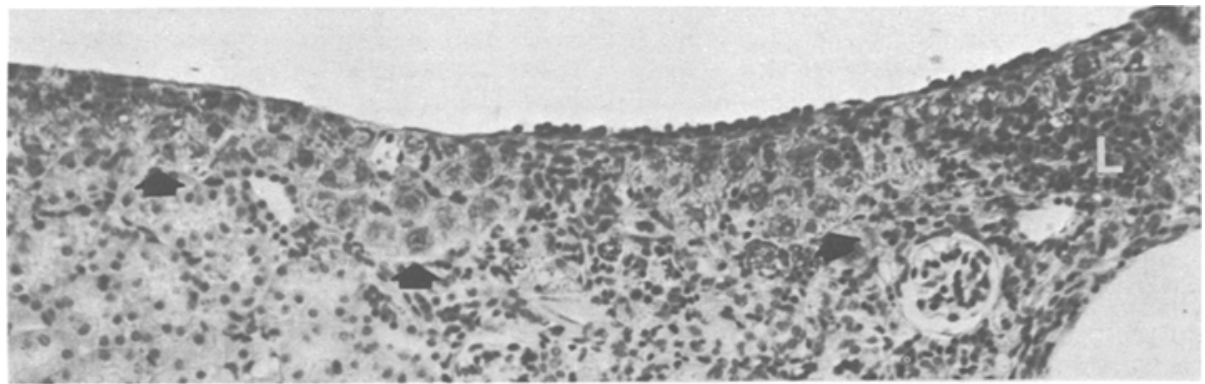

Fig. 2. B10.D2(58N) to B10 70 days after grafting. Grade 1. Lymphocytes $(L)$. Arrows indicate grafthost interface. $(200 \times)$ 


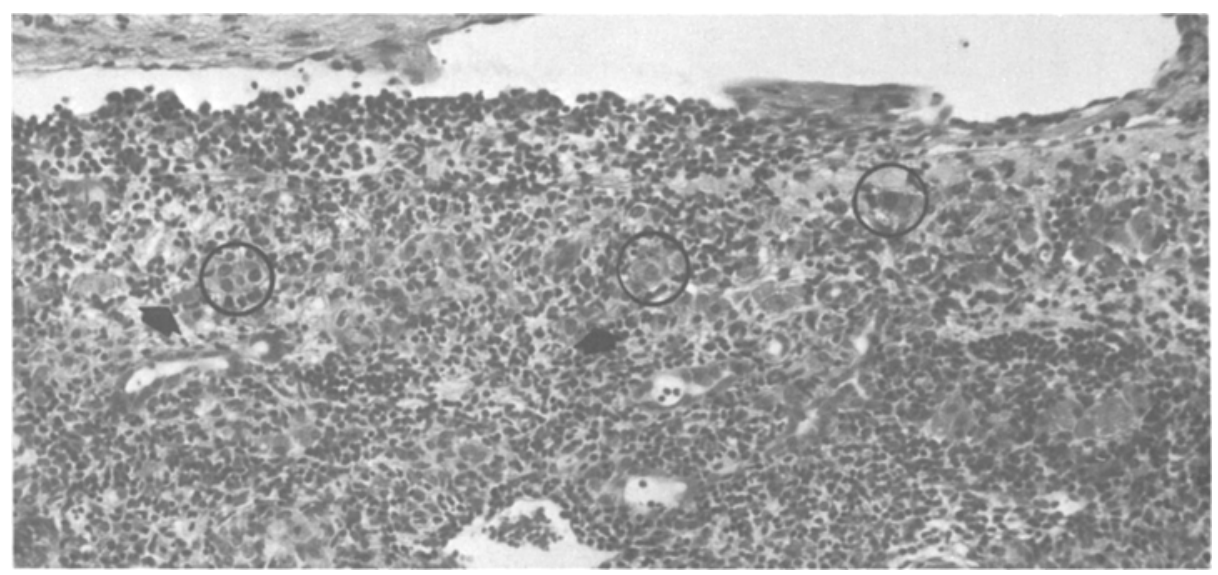

Fig. 3. B $10.129(21 \mathrm{M})$ to B10 42 days after grafting. Grade 4. Isolated degenerating hepatocytes are circled. Arrows indicate graft-host interface. $(200 \mathrm{x})$

graft information, since $H-1^{a} \rightarrow H-1^{b}$ skin grafts survive longer than $H-1^{b} \rightarrow H-1^{a}$ (Hildemann 1970).

Grafts over the $H-3^{b} \rightarrow H-3^{a}$ barrier show considerable immunological activity, beginning as early as ten days after grafting. The grafts go through a period of crisis, but by 140 days six of six grafts appear to be actively proliferating and very healthy. When an $H-4^{b} \rightarrow H-4^{a}$ barrier is involved, most grafts are in considerable immunological difficulty by 21 days after grafting and are either rejected or proliferative at 146 days. Reciprocal grafts $\left(H-4^{a} \rightarrow H-4^{b}\right)$, after passing through a crisis at 42 to 56 days, appear to recover and show proliferation at 84 days. As is shown in Table 1, grafts over the $H-8^{b} \rightarrow H-8^{a}$ barrier are almost always surviving and proliferating at 37 days.

\section{Discussion}

Liver slice-to-kidney bed grafts performed over a series of minor histocompatibility locus barriers, unlike grafts over major histocompatibility locus barriers, fail to show clear definitive times of rejection, typical of an acute rejection pattern. Instead, a chronic pattern of rejection, similar to that seen in skin grafts over these same minor barriers, is evident. A wide range of reactions are displayed in Figure 4. The ranges may express a dichotomy of cell populations with some replicate grafts surviving but showing evidence of considerable immunological reaction, some eliciting little or no immunological reactivity, and some grafts being in a state of active proliferation. The proliferative state of some of these grafts is of particular interest, since this activity serves as an additional indication of immunological enhancement, possibly because of the presence of humoral reactivity. These proliferating grafts are manifested by an increase in the hepatocyte mass, compared to syngeneic grafts. Many cells are binucleate and mitotic figures are present. In addition to an increase in the mass, proliferating hepatocytes sometimes are seen infiltrating into the adjacent kidney tissue and extending laterally beneath the kidney capsule. This type of proliferative 


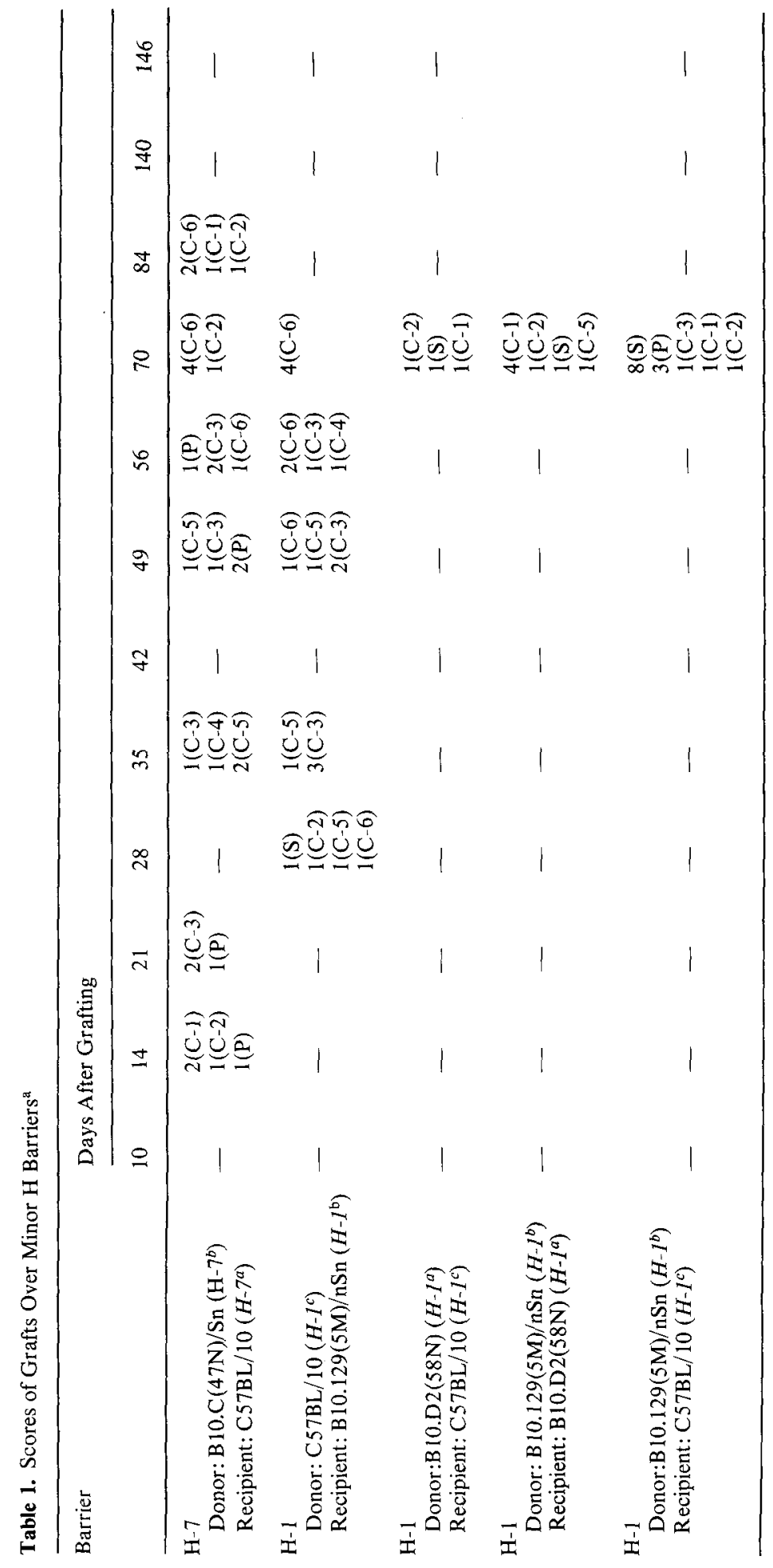




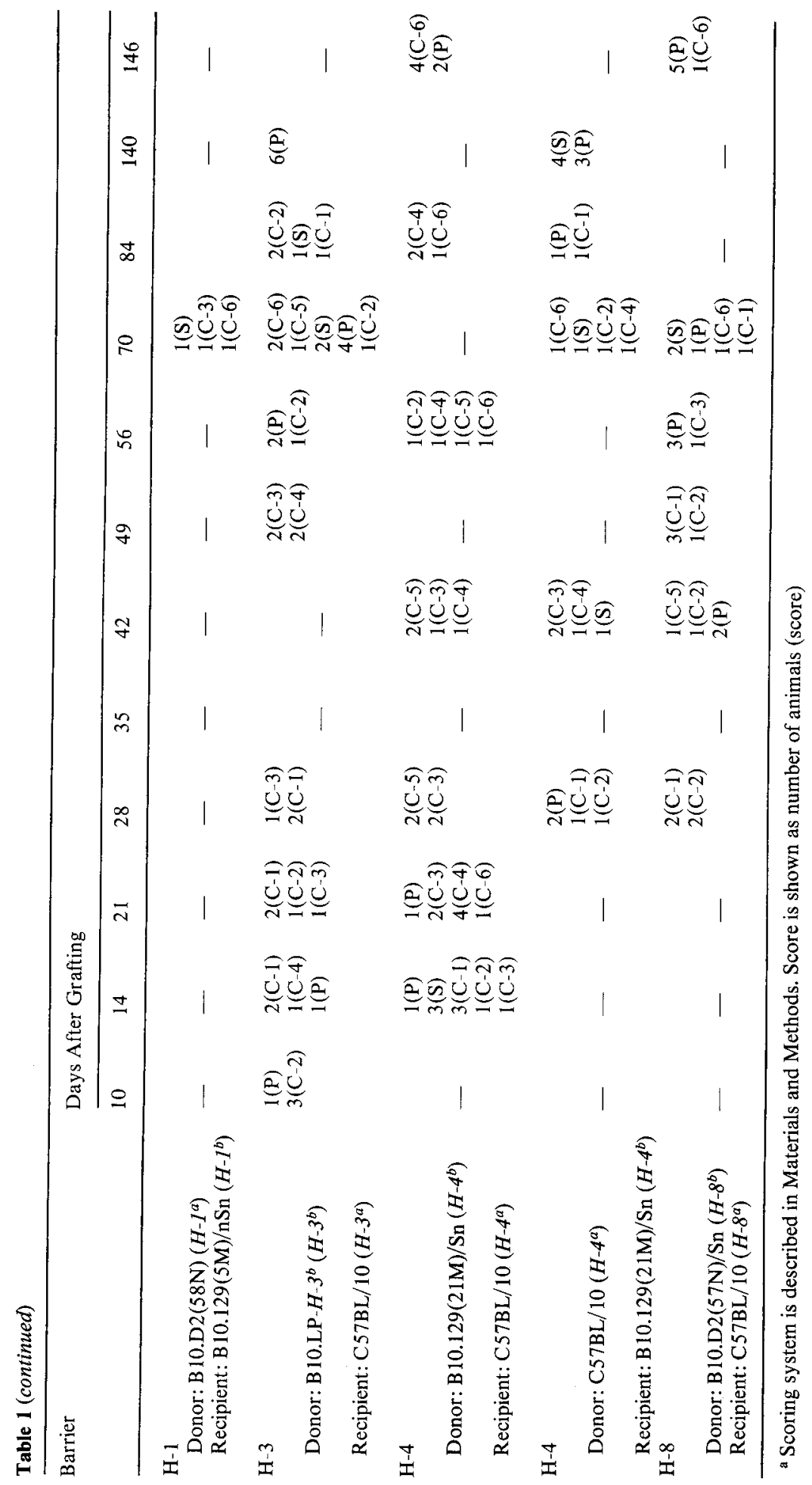



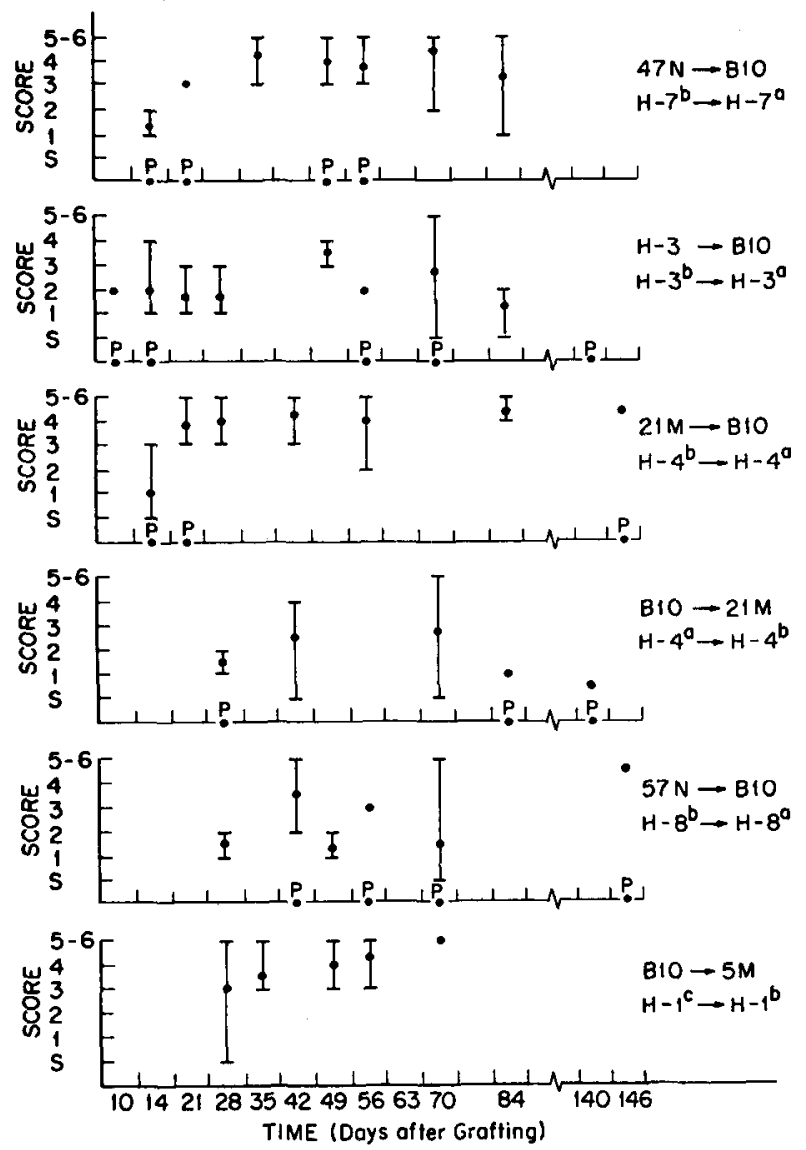

Fig. 4. Graphic representation of data presented in Table 1. The points are a numerical average of $S$ and $C$ values given the following weights: $S=1, C(1)=2, C(2)=3, C(3)=4, C(4)=5, C(5-6)=6$. The ranges shown are not necessarily continuous but may represent a dichotomy of values (e.g., a rejected group and a healthy group). Groups containing proliferative grafts are represented on the graft by $P$, but are not averaged into the final value

reactivity is seen occasionally in liver slice-to-kidney bed grafts across $\mathbf{H}-2$ subregional barriers (certain I-region and D-region barriers) which show a chronic rejection pattern (Schultz et al. 1977).

Enhancement has been reported previously for skin grafts across minor histocompatibility barriers. However, comparison between the skin graft and liver graft systems is not entirely valid because of the heterotopic nature of the latter system, the variation in results with different sizes and types of skin graft, and the extraordinary ability of liver tissue to regenerate. The skin graft data discussed here are obtained from a number of different laboratories, and so the skin grafts used varied in size and source. But since much histocompatibility system analysis is based on skin graft data, the latter system is a natural base for comparison.

Since it is impossible to view one graft over a continuous time period in the liverto-kidney bed system, it is not clear whether a single graft may go through a series of 
immunologically critical stages and then recover, or whether the time course of rejection is actually different for each graft.

Several courses would fit the observations. A graft may go from C-1 to 2, 3, 4, 5, and C- 6 in continuous sequence. There are clearly some grafts which proceed from $\mathrm{S}$ through the mild immunological reactivity of $\mathrm{C}-1$ directly to $\mathrm{P}$. These may be the grafts which, because of environmental factors, produce considerable quantities of enhancing antibody shortly after the onset of the immunological reaction. Alternatively, grafts may follow the sequence $\mathrm{C}-1$ to $\mathrm{C}-2$ to $\mathrm{P}$ or $\mathrm{C}-1$ to $\mathrm{C}-2$ to $\mathrm{C}-1$. Once a graft reaches the $\mathrm{C}-3$ stage, it may recover and proliferate or may return to the $\mathrm{C}-2$ phase with a reduction of immunological activity, recovery, and regeneration of hepatocytes. These various immunological patterns of liver grafts over minor $\mathrm{H}$ barriers are diagrammed in Figure 5 below.

Several other phenomena which do not appear in acutely rejecting grafts are revealed in liver-to-kidney grafts across minor histocompatibility barriers. Infiltrating lymphocytes are most often found scattered between degenerating hepatocytes in the graft and are rarely localized in nodules. The occasional lymphocytic nodules which are found are considerably smaller in size than those always present in the latter stages of acute graft rejection.

Polymorphonuclear leukocytes are seen in acutely rejecting grafts over major histocompatibility barriers only very early (7-10 days) after grafting. These cells are scattered throughout some grafts over minor histocompatibility barriers as late as 12 weeks after grafting. Plasma cells are also frequently present in long-term grafts over minor histocompatibility barriers and are located primarily at the grafthost interface. The presence of these cell types is further evidence for the operation of different immunological mechanisms in $\mathrm{H}-2$ and minor histoincompatible grafts of liver tissue.

Liver slice-to-kidney bed grafts failed to show radical differences in rejection patterns from those shown by skin grafts. Therefore, there is no evidence from the

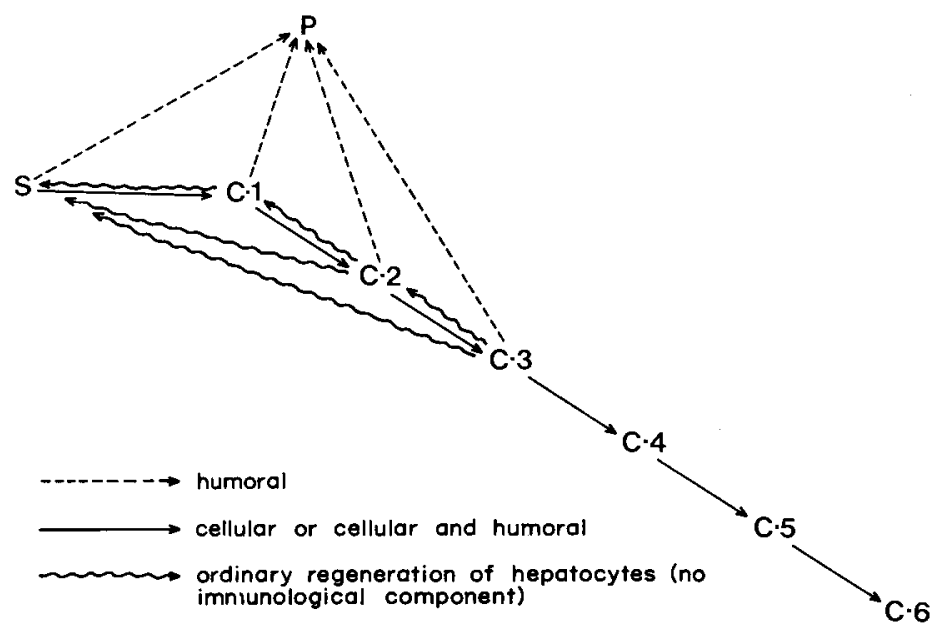

Fig. 5. 
present data that any of the minor histocompatibility barriers tested has specific importance in rejection of liver tissue. However, agreement with skin graft-rejection data is, by no means absolute. $H-7^{b}$-to- $H-7^{a}$ liver grafts and $H-I^{c}$-to- $H-1^{b}$ liver grafts show very similar rejection patterns. However, skin grafts in the former combination show mean survival times two times greater than survival times in the latter combination (Graff and Bailey 1973, Hildemann 1970).

In the $H-1^{b}$-to- $H-1^{a}$ combination, the mean survival time for skin is 25 days (Hildemann 1970). However, liver grafts in this combination are mostly healthy and surviving at 70 days after grafting. In the H-7 combination in which skin graft mean survival time is 33 days (Graff and Bailey 1973), most grafts have been completely or nearly destroyed by 70 days after grafting and the grafts do not appear to recover. $H-4^{b} \rightarrow H-4^{a}$ grafts are also in relatively poor condition at 70 days after grafting, although skin graft survival time in this combination is in the same range ( 24 days, Graff et al. 1966). Although there are a few remaining healthy grafts as late as 84 days after grafting in the $\mathrm{H}-7$ and $\mathrm{H}-4$ combinations, these barriers appear to have greater effects than the $H-I^{b}$ to $H-I^{a}$ barrier on liver graft rejection, while the three barriers have essentially identical influence on skin graft survival (Graff et al. 1966).

Skin grafts over an H-3 barrier survive only slightly longer than $\mathrm{H}-7$ barrier grafts (Graff et al. 1966). However, in the liver-to-kidney grafting systems, H-3disparate grafts, although in immunological difficulty from approximately 10 days after grafting, recover and are proliferating at 140 days after grafting.

The H-4 barrier influences liver-to-kidney grafts to nearly the same extent as skin grafts, in that the $H-4^{b} \rightarrow H-4^{a}$ barrier, which causes rejection about 20 days after grafting of skin, causes rejection or major immunological activity by 21 days in the liver system. In the reciprocal combination, however, skin survives for 119 days (Graff et al. 1966), whereas liver, after passing through a mild immunological crisis, shows active proliferative signs at 84 days after grafting.

It is clear that these minor barriers show somewhat different immunological influences on the rejection of different donor tissues as well as a clearly different immunological reaction pattern from that seen in acutely rejecting grafts.

The proliferative phenomenon observed in these grafts over minor barriers is also an intriguing one. If the proliferation is caused by production of an enhancing antibody or eliciting or production of a mitotic factor, the effect may be of considerable importance in tumor immunology. It may be that tumor growth is caused by suppression of response to certain minor histocompatibility antigens. We will continue to investigate the proliferative phenomenon as well as liver-to-kidney grafts over minor histocompatibility barriers in the presence of different $H$-2 alleles, which may yield further information on the influence of difference minor histocompatibility loci and alleles on liver graft rejection.

Acknowledgments. Supported by the Medical Research Service of the Veterans Administration and National Cancer Institute Grant CA18638.

\section{References}

Baldwin, W.M. III and Cohen, N.: "Weak" histocompatibility antigens generate functionally "strong" humoral immunity. Immunogenetics 1:33-44, 1974

Cantrell, J.L. and Hildemann, W.H.: Characteristics of disparate histocompatibility barriers in congenic strains of mice. J. Immunol. 110:629-636, 1973

Eichwald, E.J., Wetzel, B., and Lustgraff, E.C.: Genetic aspects of second-set skin grafts in mice. Transplantation 4:260-273, 1966 
French, M.E. and Batchelor, J.R.: Immunological enhancement of rat kidney graft. Lancet 1969II: 1103

Goldberg, E.H., Boyse, E.A., Bennett, D., Scheid, M., and Carswell, E.A.: Serological demonstration of $\mathrm{H}-\mathrm{Y}$ (male) antigen on mouse sperm. Nature 232:478-480, 1971

Graff, R.J., Hildemann, W.H., and Snell, G.D.: Histocompatibility genes of mice. VI. Allografts in mice congenic at various non-H-2 histocompatibility loci. Transplantation 4:425-437, 1966

Graff, R.J. and Bailey, D.W.: The non-H-2 histocompatibility loci and their antigens. Transplant. Rev. $115: 26-49,1973$

Hildemann, W.H.: Components and concepts of antigenic strength. Transplant. Rev. 3:5-21, 1970

Hildemann, W.H., Morgan, M., and Grautnick, L.: Immunogenetic components of weaker histocompatibility systems in mice. Transplant. Proc. $2: 24-31,1970$

Hilgert, I.: Variation in the type of immune response to mouse histocompatibility antigens as the function of their form. J. Immunogenet. 1:153-207, 1974

Jeekel, J.J., McKenzie, I.F.C., and Winn, J.H.: Immunological enhancement of skin graft in the mouse. J. Immunol. 108:1017-1024, 1972

Klein, J.: Strength of histocompatibility genes in mice. In E.S. Curtoni, P.L. Mattiuz and R.M. Tosi (Eds.): Histocompatibility testing 1967, pp. 21-29. Munksgaard, Copenhagen, 1967

Klein, J.: Biology of the Mouse Histocompatibility-2 Complex. Springer-Verlag, New York, 1975

Lapp, W.S. and Bliss, J.Q.: Skin graft size: Its effect on graft survival in mice incompatible at a weak locus. Transplantation 4:754-755, 1966

Mandel, M.A., Monaco, A.P., and Russell, A.P.: Destruction of spleenic transplantation antigens by a factor present in liver. J. Immunol. 95:673-682, 1965

McKenzie, I.F.C. and Snell, G.D.: Comparative immunogenicity and enhanceability of individual H$2 \mathrm{~K}$ and H-2D specificities of the murine histocompatibility-2 complex. J. Exp. Med. 138:259-277, 1973

Palm, J. and Manson, L.A.: Tissue distribution and intracellular sites of some mouse isoantigens. In J. Palm (ed.): Isoantigens and Cell Interactions, p. 21. Wistar Institute Press, Philadelphia, 1965

Schultz, J.S., Beals, T.F., and Petraitis, F.P.: Tissue graft rejection in mice. I. Contributions of H-2 and non-H-2 genetic barriers. Immunogenetics 3:85-96, 1976

Schultz, J.S., Beals, T.F., and DeMott-Friberg, R.: Tissue graft rejection in mice. II. Graft survival across $\mathrm{H}-2$ regional barriers. Immunogenetics $4: 315-325,1977$

Simonsen, M.: The clonal selection hypothesis evaluated by grafted cells reacting against their hosts. Cold Spring Harbor Symp. Quant. Biol. 32:517-523, 1967

Wettstein, P.J. and Haughton, G.: Production, testing and utility of double congenic strains of mice. Transplantation 17:513-517, 1974

Wettstein, P.J. and Haughton, G.: The genetic control of the immune response to murine histocompatibility antigen. I. The response to H-4 alloantigens. Immunogenetics 4:65-78, 1977

Wheeler, H.B., Carson, J.M., and Dammin, G.J.: Transplantation of tissue slices in mice. Ann. N.Y. Acad. Sci. 129:118-125, 1966

Zink, G.L. and Heyner, S.: The production of non-H-2 histocompatibility alloantibodies in the mouse. Immunogenetics 4:257-266, 1977

Received February 3, 1978; revised version received March 29, 1978 